J. Korean Math. Soc. 47 (2010), No. 4, pp. 691-704

DOI 10.4134/JKMS.2010.47.4.691

\title{
GORENSTEIN-INJECTORS, GORENSTEIN-FLATORS
}

\author{
Qingin Gu, Xiaosheng Zhu, and Wenping Zhou
}

\begin{abstract}
Over a ring $R$, let $P_{R}$ be a finitely generated projective right $R$-module. Then we define the $G$-injector ( $G$-projector) if $P_{R}$ preservers Gorenstein injective modules (Gorenstein projective modules), the $G$ flator if $P_{R}$ preservers Gorenstein flat modules. $G$-injector ( $G$-flator) and $G$-injector are characterized focus primarily on the cases where $R$ is a Gorenstein ring, and under this condition we also study the relations between the injector (projector, flator) and the $G$-injector ( $G$-projector, $G$-flator). Over any ring we also give the characteristics of $G$-injector $(G$ flator) by the Gorenstein injective (Gorenstein flat) dimensions of modules.
\end{abstract}

\section{Introduction}

Unless otherwise stated, throughout this paper $R$ will denote an associative ring with identity, $P_{R}$ will denote a finitely generated projective right $R$-module. ${ }_{R} P^{*}$ will denote its $R$-dual ${ }_{R} P^{*}=\operatorname{Hom}_{R}\left(P_{R}, R\right)$ and $S$ will denote its $R$-endomorphism ring $S=\operatorname{End}_{R}\left(P_{R}\right)$.

Let ${ }_{R} \mathcal{M}$ and ${ }_{S} \mathcal{M}$ be categories of all left $R$-modules and of all left $S$-modules respectively. By an $(R, S)$ adjoint triple is meant a triple $(\mathscr{G}, \mathscr{F}, \mathscr{H})$ of additive functors

$$
\mathscr{F}:{ }_{R} \mathcal{M} \rightarrow_{S} \mathcal{M} \text { and } \mathscr{G}, \quad \mathscr{H}:{ }_{S} \mathcal{M} \rightarrow_{R} \mathcal{M}
$$

such that there are natural isomorphisms

$$
\begin{aligned}
& \operatorname{Hom}_{R}(\mathscr{G}(N), M) \cong \operatorname{Hom}_{S}(N, \mathscr{F}(M)), \\
& \operatorname{Hom}_{S}(\mathscr{F}(M), N) \cong \operatorname{Hom}_{R}(M, \mathscr{H}(N))
\end{aligned}
$$

for all $M \in_{R} \mathcal{M}$ and all $N \in \in_{S} \mathcal{M}$. When ${ }_{S} P_{R}$ is a bimodule with $P_{R}$ finitely generated projective then by $[11] \mathscr{F}, \mathscr{G}, \mathscr{H}$ are naturally equivalent to the functors

$$
\begin{aligned}
& \mathscr{F}_{P}=P_{R} \otimes_{R}():_{R} \mathcal{M} \longrightarrow{ }_{S} \mathcal{M}, \\
& \mathscr{G}=\operatorname{Hom}_{R}\left(P_{R}, R\right) \otimes_{S}()::_{S} \mathcal{M} \longrightarrow_{R} \mathcal{M}, \\
& \mathscr{H}_{P}=\operatorname{Hom}_{S}\left(P_{R},\right)::_{S} \mathcal{M} \longrightarrow{ }_{R} \mathcal{M} .
\end{aligned}
$$

Received December 29, 2007; Revised August 19, 2009.

2000 Mathematics Subject Classification. 13D05, 13D02, 16E10, 16E30.

Key words and phrases. Gorenstein-injector, perfect Gorenstein-injector, Gorensteinflator, perfect Gorenstein-flator. 
Anderson [1] defined the injector, the projector and studied the characteristics of them. Moreover, he gave some conditions under which $P_{R}$ preserves the injective modules (projective modules). In [2], Miller defined the flator and generated Anderson's results. He also gave the relations among flators, injectors, and projectors. In 1995, Enochs and Jenda [6] defined the Gorenstein injective modules, Gorenstein projective modules. And defined Gorenstein flat modules in [8]. As we all know these notions generalized the usual injective, projective and flat modules.

In this paper we shall define the $G$-injector (the $G$-projector), the perfect $G$-injector, the $G$-flator and the perfect $G$-flator which generalize Anderson's injector (projector), perfect injector, and Miller's flator, perfect flator respectively.

When $P_{R}$ is a finitely generated projective generator, we know that the ring $R$ is Morita equivalent to $S=\operatorname{End}\left(P_{R}\right)$ (see [2]). It is easy to know that $P_{R}$ preserves Gorenstein injective modules (Gorenstein projective modules), and Gorenstein injective envelopes (Gorenstein projective covers). When $P_{R}$ is not a generator, $R$ and $S$ are not Morita equivalent; still, for many projective modules $P$, a considerable amount of information is often available about $S$. Here, we focus primarily on the cases where $R$ is a Gorenstein ring. We consider the conditions that $P_{R}$ preserves $G$-injective $(G$-projective) modules and $G$ flat modules. We shall show that if $P_{R}$ is a $G$-injector ( $G$-flator), then $P_{R} \otimes-$ preservers modules with finite Gorenstein injective (Gorenstein flat) dimensions, that is, if $N$ is a left $R$-module of finite Gorenstein injective (Gorenstein flat) dimension, then ${ }_{S} P_{R} \otimes N$ is a left $S$-module of finite Gorenstein injective (Gorenstein flat) dimension. We also give the relation between the $G$-injector ( $G$-flator) and the Gorenstein injective dimensions of modules.

\section{Preliminaries}

In this section we shall recall some definitions which we use later.

Definition 1.1 (Gorenstein injective module). A left $R$-module $M$ is said to be Gorenstein injective if and only if there is an exact sequence

$$
\cdots \rightarrow_{R} E_{1} \rightarrow_{R} E_{0} \rightarrow_{R} E^{0} \rightarrow_{R} E^{1} \rightarrow \cdots
$$

of injective left $R$-modules such that $M=\operatorname{Ker}\left({ }_{R} E^{0} \rightarrow_{R} E^{1}\right)$ and such that for any injective left $R$-module $E, \operatorname{Hom}(E,-)$ leaves the complex above exact. Dually, we can define the Gorenstein projective module.

A left $R$-module $N$ is said to be Gorenstein flat (or $G$-flat) if there exists an $(\mathcal{I} n j \otimes-)$-exact exact sequence

$$
\cdots \rightarrow_{R} F_{1} \rightarrow_{R} F_{0} \rightarrow_{R} F^{0} \rightarrow_{R} F^{1} \rightarrow \cdots
$$

of flat $R$-modules such that $N=\operatorname{Ker}\left({ }_{R} F^{0} \rightarrow_{R} F^{1}\right)$ (see [7]). The above exact sequence is called a complete flat resolution. 
Definition 1.2 (Strongly Gorenstein injective module). If all injective (resp., projective) modules and homomorphisms of the complete injective (resp., projective) resolution in Definition 1.1 are the same, then $M$ is called strongly Gorenstein projective (resp., injective).

If all flat modules and homomorphisms of the complete flat resolution in Definition 1.1 are the same, then $N$ is called strongly Gorenstein flat (see [3]).

Definition 1.3 (Injector and flator). We call ${ }_{S} P_{R}$ an injector (projector, flator) in case $F_{P}=P_{R} \otimes-$ preserves injective (projective, flat) modules where $S=$ $\operatorname{End}\left(P_{R}\right)$, that is, $F_{P}(M)$ is $S$-injective (projective, flat) whenever $M$ is $R$ injective (projective, flat) (see [1] and [10]).

Definition 1.4 (Gorenstein injective preenvelope). A Gorenstein injective preenvelope of an $R$-module $M$, we mean a morphism $\varphi:_{R} M \rightarrow_{R} G$ where $G$ is a Gorenstein injective module such that for any morphism $f:_{R} M \rightarrow_{R} G^{\prime}$ with $G^{\prime}$ is Gorenstein injective, there is a $g:_{R} F \rightarrow_{R} F^{\prime}$ such that $g \circ \varphi=f$. Dually, we can define the Gorenstein flat (Gorenstein projective) precover (see [7]).

Definition 1.5 (Gorenstein injective dimension). For an $R$-module $M$ we said its Gorenstein injective dimension is equal to or less than $n$ if it has a Gorenstein injective resolution whose length is equal to or less than $n$, we denote it by $\operatorname{Gid}_{R} M \leq n$ (see [9]).

Definition 1.6 (Copure injective). An $R$-module $M$ is said to be copure injective (copure flat) if $\operatorname{Ext}_{R}^{1}(E, M)=0\left(\operatorname{Tor}_{1}^{R}(E, M)=0\right)$ for any injective $R$-module $E$. Also, $M$ is said to be strongly copure injective (strongly copure flat) if $\operatorname{Ext}_{R}^{i}(E, M)=0\left(\operatorname{Tor}_{i}^{R}(E, M)=0\right)$ for any injective $R$-module $E$ and any $i>0$ (see [5]).

Definition $1.7(T(p))$. In general $\varphi:_{R} P_{S}^{*} \otimes_{S} P_{R}$ is not an isomorphism, the image of $\varphi$ is a two sided ideal $T=T(P)$ of $R$ called the trace ideal of $P$, thus $T=\operatorname{Im} \varphi=\Sigma \operatorname{Im} f \quad\left(f \in P^{*}\right)($ see $[1])$.

Remark. By $\mathcal{I}(R)$ we denote the class of injective left $R$-modules, and by $\widetilde{\mathcal{G I}}(R), \widetilde{\mathcal{G F}}(R)$ we denote the classes of all $R$-modules with finite Gorenstein injective, flat dimensions respectively. By $\mathcal{I}(S)$ we denote the class of injective left $S$-modules, and by $\widetilde{\mathcal{G I}}(S), \widetilde{\mathcal{G F}}(S)$ we denote the classes of all $S$-modules with finite Gorenstein injective, flat dimensions respectively.

\section{Gorenstein-injectors}

Let $P_{R}$ be a finitely generated projective module, and $S=\operatorname{End}\left(P_{R}\right)$. We call $P_{R}$ a $G$-injector if $P_{R}$ preservers Gorenstein injective modules, that is, for any Gorenstein injective left $R$-module ${ }_{R} M,{ }_{S} P_{R} \otimes_{R} M$ is a Gorenstein injective left $S$-module.

Proposition 2.1. Let ${ }_{S} P_{R}$ be an injector. If ${ }_{R} P_{S}^{*} \otimes_{S}-$ preserves injective modules, then $P_{R}$ is a G-injector. 
Proof. Assume that $M$ is a Gorenstein injective left $R$-module, then there exists a $\operatorname{Hom}(\mathcal{I}(R),-)$-exact exact sequence

$$
\cdots \rightarrow E_{2} \rightarrow E_{1} \rightarrow E_{0} \rightarrow E^{0} \rightarrow E^{1} \rightarrow E^{2} \rightarrow \cdots
$$

of injective left $R$-modules such that

$$
M=\operatorname{Ker}\left(E^{0} \rightarrow E^{1}\right) .
$$

Applying $P_{R} \otimes-$ to the above exact sequence, we get an exact sequence:

$$
\cdots \rightarrow P_{R} \otimes_{R} E_{1} \rightarrow P_{R} \otimes_{R} E_{0} \rightarrow P_{R} \otimes_{R} E^{0} \rightarrow P_{R} \otimes_{R} E^{1} \rightarrow \cdots .
$$

Since $P_{R}$ is an injector each $P_{R} \otimes_{R} E_{i}$ and each $P_{R} \otimes_{R} E^{i}$ are injective. Clearly, $P_{R} \otimes_{R} M=\operatorname{Ker}\left(P_{R} \otimes_{R} E^{0} \rightarrow P_{R} \otimes_{R} E^{1}\right)$. Note that

$$
\operatorname{Hom}_{S}\left(E^{\prime}, P_{R} \otimes E\right) \cong \operatorname{Hom}_{R}\left({ }_{R} P_{S}^{*} \otimes E^{\prime}, E\right)
$$

for any left $S$-module $E^{\prime}$ and any left $R$-module $E$, and ${ }_{R} P_{S}^{*} \otimes-$ preserves injective modules; then $(1)$ is a $\operatorname{Hom}(\mathcal{I}(S),-$ )-exact exact sequence of injective left $S$-modules. Thus $P_{R}$ is a $G$-injector.

Lemma 2.2. Let ${ }_{S} P_{R}$ be an $(R, S)$-bimodule with ${ }_{S} P$ projective and $P_{R}$ finitely generated projective. If $R$ is Gorenstein, then $S=\operatorname{End}\left(P_{R}\right)$ is Gorenstein.

Proof. Since $R$ is a Gorenstein ring, $S$ is Notherian by [1], [7, Theorem 9.1.17] gives that $P_{R}$ has finite injective dimension. Now we assume $i d\left(P_{R}\right)=n<\infty$, That is, there exists an injective resolution of $P_{R}$ :

$$
0 \rightarrow{ }_{S} P_{R} \rightarrow E_{R}^{0} \rightarrow E_{R}^{1} \rightarrow E_{R}^{2} \rightarrow \cdots \rightarrow E_{R}^{n} \rightarrow 0 .
$$

Applying $\operatorname{Hom}_{R}\left({ }_{S} P_{R},-\right)$ to this resolution, we get

$$
0 \rightarrow \operatorname{Hom}_{S}\left({ }_{S} P_{R},{ }_{S} P_{R}\right) \rightarrow \operatorname{Hom}_{S}\left({ }_{S} P_{R}, E_{R}^{0}\right) \rightarrow \cdots \rightarrow \operatorname{Hom}_{S}\left({ }_{S} P_{R}, E_{R}^{n}\right) \rightarrow 0
$$

as each $\operatorname{Hom}_{S}\left({ }_{S} P_{R}, E_{R}^{i}\right)$ is injective as an $S$-module. So $S$ has finite selfinjective dimension. Thus $S$ is Gorenstein.

Corollary 2.3. Let ${ }_{S} P_{R}$ be an $(R, S)$-bimodule with ${ }_{S} P$ projective and $P_{R}$ finitely generated projective. If $R$ is $n$-Gorenstein, then $S=\operatorname{End}\left(P_{R}\right)$ is $m$ Gorenstein $(m \leq n)$.

Proposition 2.4. Let ${ }_{S} P_{R}$ be an $(R, S)$-bimodule with ${ }_{S} P$ projective and $P_{R}$ finitely generated projective, and let $R$ be a Gorenstein ring. If ${ }_{S} P_{R}$ is a $G$ injector, then $P_{R}$ is an injector.

Proof. Let $E_{R}$ be an injective left $R$-module. Then ${ }_{S} P_{R} \otimes_{R} E$ is a Gorenstein injective left $S$-module. Since $R$ is Gorenstein and ${ }_{R} E$ is injective by [7, Theorem 9.1.17], we know that ${ }_{R} E$ has finite projective dimension. Assume that $p d_{R}(E)=n$. So there exists a projective resolution of $E$

$$
0 \rightarrow_{R} P_{n} \rightarrow \cdots \rightarrow_{R} P_{2} \rightarrow_{R} P_{1} \rightarrow_{R} E \rightarrow 0 .
$$


Since ${ }_{S} P_{R}$ is left $S$-projective, ${ }_{S} P_{R} \otimes_{R} P_{i}$ is projective by [1, Theorem 3.1 . Applying ${ }_{S} P_{R} \otimes-$ to $(2)$, we get a projective resolution of ${ }_{R} E$

(3) $0 \rightarrow_{S} P_{R} \otimes_{R} P_{n} \rightarrow \cdots \rightarrow_{S} P_{R} \otimes_{R} P_{2} \rightarrow_{S} P_{R} \otimes_{R} P_{1} \rightarrow_{S} P_{R} \otimes_{R} E \rightarrow 0$.

Then ${ }_{S} P_{R} \otimes_{R} E$ has finite projective dimension, and so the injective dimension of ${ }_{S} P_{R} \otimes_{R} E$ is finite by [7, Theorem 9.1.17]. Thus ${ }_{S} P_{R} \otimes_{R} E$ is injective by [7, Proposition 10.1.2].

Lemma 2.5. Let $R$ be Gorenstein, and let $M$ be a Gorenstein injective $R$ module. Then there exists an exact sequence $0 \rightarrow K \rightarrow E \rightarrow M \rightarrow 0$ such that $E$ is injective and $K$ is Gorenstein injective.

Proof. See [13, Lemma 5.4.3].

Theorem 2.6. Let $R$ be an $n$-Gorenstein ring, let ${ }_{S} P_{R}$ be an $(R, S)$-bimodule with ${ }_{S} P$ projective and $P_{R}$ finitely generated projective. If $P_{R}$ is an injector, then $P_{R}$ is a $G$-injector.

Proof. Suppose that $M$ is a Gorenstein injective left $R$-module. By Lemma 2.5, we can construct an exact sequence

$$
\cdots \rightarrow E_{i+1} \rightarrow E_{i} \rightarrow \cdots \rightarrow E_{1} \rightarrow E_{0} \rightarrow M \rightarrow 0
$$

in which each $E_{i}$ is injective and each $K_{i}=\operatorname{Coker}\left(E_{i+1} \rightarrow E_{i}\right)(i \geq 1)$ is Gorenstein injective. Consider the short exact sequences $0 \rightarrow_{R} K_{1} \rightarrow_{R} E_{0} \rightarrow_{R}$ $M \rightarrow 0$ and $0 \rightarrow_{R} K_{i+1} \rightarrow_{R} E_{i} \rightarrow_{R} K_{i} \rightarrow 0$ for all $i$.

Applying ${ }_{S} P_{R} \otimes-$ to (4), we have the short exact sequences

$$
0 \rightarrow_{S} P_{R} \otimes_{R} K_{1} \rightarrow_{S} P_{R} \otimes_{R} E_{0} \rightarrow_{S} P_{R} \otimes_{R} M \rightarrow 0
$$

and

$$
0 \rightarrow_{S} P_{R} \otimes_{R} K_{i+1} \rightarrow_{S} P_{R} \otimes_{R} E_{i} \rightarrow_{S} P_{R} \otimes_{R} K_{i} \rightarrow 0
$$

for all $i$. Since $P_{R} \otimes_{R} E_{i}$ is injective, we have the following exact sequence:

$\cdots \rightarrow{ }_{S} P_{R} \otimes E_{i+1} \rightarrow{ }_{S} P_{R} \otimes E_{i} \rightarrow \cdots \rightarrow_{S} P_{R} \otimes E_{1} \rightarrow_{S} P_{R} \otimes E_{0} \rightarrow_{S} P_{R} \otimes{ }_{R} M \rightarrow 0$.

By Corollary 2.3, $S$ is $m$-Gorenstein. [7, Theorem 10.1.13] gives that each ${ }_{S} P_{R} \otimes K_{i}$ is Gorenstein injective for $i \geq m-1$. By [7, Theorem 10.1.4] and the short exact sequence

$$
0 \rightarrow_{S} P_{R} \otimes K_{m-1} \rightarrow_{S} P_{R} \otimes E_{m-2} \rightarrow_{S} P_{R} \otimes K_{m-2} \rightarrow 0,
$$

we conclude that ${ }_{S} P_{R} \otimes K_{m-2}$ is Gorenstein injective. By repeating this argument for the other short exact sequences we have ${ }_{S} P_{R} \otimes M$ is a Gorenstein injective left $S$-module.

Proposition 2.7. Let ${ }_{S} P_{R}$ be a finitely generated projective left $R$-module. If $T(P)$ is right $R$-flat and ${ }_{R} P_{S}^{*} \otimes-$ preserves injective modules, then $P_{R}$ is a G-injector.

Proof. Since $T(P)$ is right $R$-flat, $P_{R}$ is an injector by [1, Theorem 2.2]. So $P_{R}$ is a $G$-injector by Proposition 2.1. 
A left $R$-module $M$ is Gorenstein injective if and only if $\operatorname{Ext}_{R}^{i}(\mathcal{I}(R), M)=0$ and $M$ admits a proper left injective resolution by [9]. But if $\operatorname{Ext}_{R}^{i}(\mathcal{I}(R), M)=$ 0 , then $M$ is strongly copure injective. Using the relations between Gorenstein injective modules and copure injective modules, we get the following results.

Proposition 2.8. Let $P_{R}$ be an injector, and let $P_{R} \otimes_{R} M$ be copure injective for any Gorenstein injective left $R$-module $M$. Then $P_{R}$ is a $G$-injector.

Proof. Assume that $M$ is a Gorenstein injective left $R$-module. So there exists a $\operatorname{Hom}(\mathcal{I}(R),-)$-exact exact sequence

$$
\cdots \rightarrow_{R} E_{1} \rightarrow_{R} E_{0} \rightarrow_{R} E^{0} \rightarrow_{R} E^{1} \rightarrow \cdots
$$

of injective $R$-modules such that $M=\operatorname{Ker}\left(E^{0} \rightarrow E^{1}\right)$. Applying ${ }_{S} P_{R} \otimes-$ to this exact sequence, we get the following exact sequence of copure injective $S$-modules

$$
\cdots \rightarrow_{S} P_{R} \otimes_{R} E_{1} \rightarrow_{S} P_{R} \otimes_{R} E_{0} \rightarrow_{S} P_{R} \otimes_{R} E^{0} \rightarrow_{S} P_{R} \otimes_{R} E^{1} \rightarrow \cdots,
$$

where ${ }_{S} P_{R} \otimes_{R} M=\operatorname{Ker}\left({ }_{S} P_{R} \otimes_{R} E^{0} \rightarrow_{S} P_{R} \otimes_{R} E^{1}\right)$. By using the hypothesis for any injective $S$-module ${ }_{S} E$, the functor $\operatorname{Hom}_{S}\left({ }_{S} E,-\right)$ makes the above exact sequence exact. Thus ${ }_{S} P_{R} \otimes_{R} M$ is Gorenstein injective.

Proposition 2.9. Let ${ }_{S} P_{R}$ be an injector and ${ }_{S} P_{R} \otimes_{R} M$ be copure injective for all Gorenstein injective $R$-modules $M$. If a left $R$-module $N \in \widetilde{\mathcal{G I}}(R)$, then the left $S$-module ${ }_{S} P_{R} \otimes_{R} N \in \widetilde{\mathcal{G I}}(S)$.

Proof. We proceed by induction on $\operatorname{Gid}_{R}(N)=n$. If $n=0$, then $N$ is Gorenstein injective. Hence the result follows by Proposition 2.8. Now suppose inductively that the result has been proved for all values smaller than $n$, and so we prove it for $n$. As $\operatorname{Gid}_{R}(N)=n$, there is an exact sequence $0 \rightarrow N \rightarrow M \rightarrow C \rightarrow 0$ of $R$-modules such that $M$ is Gorenstein injective and $\operatorname{Gid}_{R}(C) \leq n-1$ by [9, Proposition 2.18]. Applying ${ }_{S} P_{R} \otimes_{R}-$ to the above exact sequence, we get the following exact sequence of $S$-modules.

$$
0 \rightarrow_{S} P_{R} \otimes_{R} N \rightarrow_{S} P_{R} \otimes_{R} M \rightarrow_{S} P_{R} \otimes_{R} C \rightarrow 0 .
$$

Then we can use the induction hypothesis for $C$ and conclude that ${ }_{S} P_{R} \otimes_{R} C$ has finite Gorenstein injective dimension. By Proposition 2.8 the left $S$-module ${ }_{S} P_{R} \otimes M$ is Gorenstein injective. Then the proceeding exact sequence implies that ${ }_{S} P_{R} \otimes N$ has finite Gorenstein injective dimension.

Corollary 2.10. Let ${ }_{S} P_{R}$ be a finitely generated projective left $R$-module, and let ${ }_{S} P_{R}$ be a $G$-injector. If a left $R$-module $N \in \widetilde{\mathcal{G I}}(R)$, then the ${ }_{S} P_{R} \otimes_{R} N \in$ $\widetilde{\mathcal{G I}}(S)$.

Next we shall study the relation between the $G$-injectors and the Gorenstein injective dimensions of the left $R$-modules. 
Theorem 2.11. Let ${ }_{S} P_{R}$ be a finitely generated projective right $R$-module, and $S=\operatorname{End}\left(P_{R}\right)$. Then ${ }_{S} P_{R}$ is a $G$-injector $\Leftrightarrow \operatorname{Gid}_{S}\left({ }_{S} P_{R} \otimes_{R} M\right) \leq \operatorname{Gid}_{R}\left({ }_{R} M\right)$ for any left $R$-module $M$.

Proof. ( $\Longrightarrow$ ) Let $M$ be a left $R$-module. Then $M$ has a Gorenstein injective resolution:

$$
0 \rightarrow M \rightarrow_{R} E^{0} \rightarrow_{R} E^{1} \rightarrow_{R} E^{2} \rightarrow \cdots .
$$

Applying ${ }_{S} P_{R} \otimes-$ to this resolution, we get the following exact sequence:

$$
0 \rightarrow_{S} P_{R} \otimes_{R} M \rightarrow_{S} P_{R} \otimes_{R} E^{0} \rightarrow_{S} P_{R} \otimes_{R} E^{1} \rightarrow \cdots .
$$

Where each ${ }_{S} P_{R} \otimes_{R} E^{i} \quad(i \geq 0)$ is a Gorenstein injective left $S$-module as $P_{R}$ is a $G$-injector. So $\operatorname{Gid}_{S}\left({ }_{S} P_{R} \otimes_{R} M\right) \leq \operatorname{Gid}_{R}\left({ }_{R} M\right)$.

$(\Longleftarrow)$ Suppose that $\operatorname{Gid}_{S}\left({ }_{S} P_{R} \otimes_{R} M\right) \leq \operatorname{Gid}_{R}\left({ }_{R} M\right)$. Let $M$ be a Gorenstein injective left $R$-module. Then $\operatorname{Gid}_{R}\left({ }_{R} M\right)=0$, so $\operatorname{Gid}_{S}\left({ }_{S} P_{R} \otimes_{R} M\right)=0$, and thus ${ }_{S} P_{R} \otimes_{R} M$ is a Gorenstein injective module. Therefore, ${ }_{S} P_{R}$ is a Gorenstein injector.

From the above theorem a natural problem is: under what conditions does the $\operatorname{Gid}_{S}\left({ }_{S} P_{R} \otimes M\right)=\operatorname{Gid}_{R}\left({ }_{R} M\right)$ hold? Next we shall discuss this problem. For this reason, we shall give the following new definition.

Definition 2.12 (Bi-G-injector). We call $P_{R}$ a Bi- $G$-injector if ${ }_{S} P_{R}$ is a $G$ injector and for any left $R$-module ${ }_{R} M,{ }_{S} P_{R} \otimes_{R} M$ is Gorenstein injective then ${ }_{R} M$ is Gorenstein injective. When $P_{R}$ is a finitely generated projective generator, $P_{R}$ is a Bi-G-injector.

Theorem 2.13. Let ${ }_{S} P_{R}$ be a finitely generated projective left $R$-module. Then ${ }_{S} P_{R}$ is a Bi-G-injector $\Leftrightarrow \operatorname{Gid}_{S}\left({ }_{S} P_{R} \otimes_{R} M\right)=\operatorname{Gid}_{R}\left({ }_{R} M\right)$ for any left $R$-module $M$.

Proof. $(\Longleftarrow)$ It is trivial.

$(\Longrightarrow)$ Suppose that $P_{R}$ is a Bi-injector. Let $M$ be a left $R$-module. Then $\operatorname{Gid}_{S}\left({ }_{S} P_{R} \otimes_{R} M\right) \leq \operatorname{Gid}_{R}\left({ }_{R} M\right)$ by Theorem 2.11.

Next we shall show $\operatorname{Gid}_{S}\left({ }_{S} P_{R} \otimes_{R} M\right) \geq \operatorname{Gid}_{R}\left({ }_{R} M\right)$.

If $\operatorname{Gid}\left({ }_{S} P_{R} \otimes_{R} M\right)=\infty$. Then it is trivial.

If $\operatorname{Gid}\left({ }_{S} P_{R} \otimes_{R} M\right)=n<\infty$. Then there exists a Gorenstein injective resolution of $M$ :

$$
0 \rightarrow_{R} M \rightarrow_{R} E^{0} \rightarrow_{R} E^{1} \rightarrow_{R} E^{2} \rightarrow \cdots \rightarrow_{R} E^{n},
$$

where each $E^{i}(i \geq 0)$ is Gorenstein injective. Note that ${ }_{S} P_{R}$ is a $\mathrm{Bi}-G$-injector and finitely generated projective right $R$-module, then

$$
0 \rightarrow_{S} P_{R} \otimes_{R} M \rightarrow_{S} P_{R} \otimes_{R} E^{0} \rightarrow_{S} P_{R} \otimes_{R} E^{1} \rightarrow \cdots \rightarrow P_{R} \otimes_{R} E^{n}
$$

is a Gorenstein injective resolution of ${ }_{S} P_{R} \otimes_{R} M$. Since $\operatorname{Gid}\left({ }_{S} P_{R} \otimes_{R} M\right)=$ $n<\infty$, then $\operatorname{Im}\left({ }_{S} P_{R} \otimes_{R} E^{n-2} \rightarrow_{S} P_{R} \otimes_{R} E^{n-1}\right)$ is Gorenstein injective, but ${ }_{S} P_{R} \otimes_{R} \operatorname{Im}\left(E^{n-3} \rightarrow E^{n-2}\right) \cong \operatorname{Im}\left({ }_{S} P_{R} \otimes_{R} E^{n-2} \rightarrow_{S} P_{R} \otimes_{R} E^{n-1}\right)$ and 
${ }_{S} P_{R}$ is a Bi-G-injector, hence $\operatorname{Im}\left(E^{n-3} \rightarrow E^{n-2}\right)$ is Gorenstein injective. So $\operatorname{Gid}\left({ }_{R} M\right) \leq n$.

In [1] F. W. Anderson defined the perfect injector. For a finitely generated $R$-module $P_{R},{ }_{S} P_{R}$ is called a perfect injector if $P_{R} \otimes_{R}-$ preserves injective envelopes. We call $P_{R}$ is a perfect $G$-injector if $P_{R} \otimes_{R}-$ preserves Gorenstein injective preenvelopes. Of course, every perfect $G$-injector is a $G$-injector.

Theorem 2.14. Let $R$ be a Gorenstein ring, and let ${ }_{S} P_{R}$ be an $(R, S)$-bimodule with $P_{R}$ finitely generated projective and ${ }_{S} P$ projective, $S=\operatorname{End}\left(P_{R}\right)$. Then $P_{R}$ is an injector $\Leftrightarrow P_{R}$ is a perfect $G$-injector.

Proof. ( $\Longleftarrow)$ By Proposition 2.4.

$(\Longrightarrow)$ Suppose that $P_{R}$ is an injector. Then $P_{R}$ is a $G$-injector by Theorem 2.6. First, we shall prove $\operatorname{Hom}_{R}\left({ }_{S} P_{R},-\right)$ preserves Gorenstein injective left $S$-modules. Let ${ }_{S} M$ be a Gorenstein injective left $S$-module, then there is a complete resolution of injective modules

(4) $\mathbb{M}_{1}=\cdots \rightarrow_{S} E_{2} \rightarrow_{S} E_{1} \rightarrow_{S} E_{0} \rightarrow_{S} E^{0} \rightarrow_{S} E^{1} \rightarrow_{S} E^{1} \rightarrow_{S} E^{2} \rightarrow \cdots$,

where ${ }_{S} M=\operatorname{Ker}\left({ }_{S} E^{0} \rightarrow_{S} E^{1}\right)$. Applying $\operatorname{Hom}_{S}\left({ }_{S} P_{R},-\right)$ to (4) we get the following exact sequence:

(5)

$$
\mathbb{M}_{2}=\cdots \rightarrow \operatorname{Hom}\left({ }_{S} P_{R},{ }_{S} E_{0}\right) \rightarrow \operatorname{Hom}\left({ }_{S} P_{R},{ }_{S} E^{0}\right) \rightarrow \operatorname{Hom}\left({ }_{S} P_{R},{ }_{S} E^{1}\right) \rightarrow \cdots .
$$

Since ${ }_{S} P_{R}$ is $R$-projective and ${ }_{S} E_{i}$ is injective, by [12, Theorem 3.44] each $\operatorname{Hom}_{s}\left({ }_{S} P_{R}, S E_{i}\right)$ is an injective $R$-module. Note that

$$
\operatorname{Hom}_{R}\left({ }_{R} E^{\prime}, \operatorname{Hom}\left({ }_{S} P_{R},{ }_{S} E_{i}\right)\right) \cong \operatorname{Hom}_{S}\left({ }_{S} P_{R} \otimes_{R} E^{\prime}{ }_{S} E_{i}\right)
$$

for any left $R$-module $E^{\prime}$ and any left $S$-module. For each injective $R$-module $E^{\prime},{ }_{S} P_{R} \otimes_{R} E^{\prime}$ is an injective $S$-module as ${ }_{S} P_{R}$ preserves injective modules. Then the sequence $\operatorname{Hom}_{S}\left({ }_{S} P_{R} \otimes_{R} E^{\prime}, \mathbb{M}_{1}\right)$ is exact, this gives that the sequence $\operatorname{Hom}_{R}\left({ }_{R} E^{\prime}, \mathbb{M}_{2}\right)$ is also exact. So $\operatorname{Hom}_{S}\left({ }_{S} P_{R},-\right)$ preserves Gorenstein injective modules.

Next, we shall show that ${ }_{S} P_{R}$ preserves Gorenstein injective preenvelopes. Let ${ }_{R} M \rightarrow_{R} G$ be a Gorenstein injective preenvelope of ${ }_{R} M$. Since ${ }_{S} P_{R}$ is a $G$-injector then ${ }_{S} P_{R} \otimes_{R} G$ is Gorenstein injective. For any Gorenstein injective left $S$-module ${ }_{S} G^{\prime}$ we get the commutative diagram:

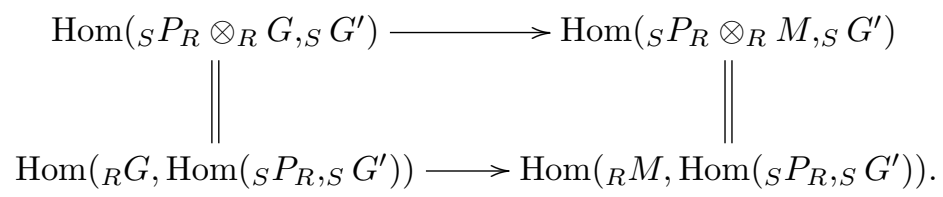

$\operatorname{Hom}\left({ }_{S} P_{R},-\right)$ preserves Gorenstein injective modules. So

$$
\operatorname{Hom}\left({ }_{R} G, \operatorname{Hom}\left({ }_{S} P_{R},{ }_{S} G^{\prime}\right)\right) \rightarrow \operatorname{Hom}\left({ }_{R} M, \operatorname{Hom}\left({ }_{S} P_{R},{ }_{S} G^{\prime}\right)\right) \rightarrow 0
$$

is exact. 
By the commutative diagram (6) we know that

$$
\operatorname{Hom}_{S}\left({ }_{S} P_{R} \otimes G, G^{\prime}\right) \rightarrow \operatorname{Hom}_{S}\left({ }_{S} P_{R} \otimes M, G^{\prime}\right)
$$

is surjective. So ${ }_{S} P_{R} \otimes_{R} M \rightarrow{ }_{S} P_{R} \otimes_{R} G$ is a Gorenstein injective preenvelope.

Remark 2.15. Dually, one can also define the Gorenstein projector. We call $P_{R}$ a $G$-projector if $P_{R}$ preserves Gorenstein projective modules, that is, for any Gorenstein projective left $R$-module ${ }_{R} M,{ }_{S} P_{R} \otimes_{R} M$ is a Gorenstein projective left $S$-module. All the results, concerning Gorenstein injective, have a Gorenstein projective counterpart.

\section{3. $G$-flator}

Let $P_{R}$ be a finitely generated projective right $R$-module, and $S=\operatorname{End}\left(P_{R}\right)$. We call $P_{R}$ a $G$-flator if $P_{R}$ preservers Gorenstein flat modules, that is, for any Gorenstein flat module ${ }_{R} M,{ }_{S} P_{R} \otimes_{R} M$ is a Gorenstein flat left $S$-module.

Proposition 3.1. Let ${ }_{S} P_{R}$ be finitely generated projective and $-{ }_{S} \otimes_{S} P_{R}$ preserve injective right $S$-modules, $S=\operatorname{End}\left(P_{R}\right)$. If $P_{R}$ is a flator, then $P_{R}$ is a $G$-flator.

Proof. Let $F$ be a Gorenstein flat module. By the definition of the Gorenstein flat module, there exists an $I_{R} \otimes-\left(I_{R}\right.$ is an arbitrary injective right $R$-module) complete flat resolution

$$
\mathbb{F}=\cdots \rightarrow_{R} F_{1} \rightarrow_{R} F_{0} \rightarrow_{R} F^{0} \rightarrow_{R} F^{1} \rightarrow \cdots
$$

such that $F=\operatorname{Ker}\left({ }_{R} F^{0} \rightarrow_{R} F^{1}\right)$. Applying $P_{R} \otimes_{R}-$ to $(7)$, we get a resolution of flat modules

(8) $\quad \cdots \rightarrow_{S} P_{R} \otimes_{R} F_{1} \rightarrow_{S} P_{R} \otimes_{R} F_{0} \rightarrow_{S} P_{R} \otimes_{R} F^{0} \rightarrow_{S} P_{R} \otimes_{R} F^{1} \rightarrow \cdots$.

By the hypothesis, for each injective $S$-module $I_{S}, I_{S} \otimes_{S} P_{R}$ is an injective right $R$-module. So $\left(I_{S} \otimes_{S} P_{R}\right) \otimes_{R} \mathbb{F}$ is exact by the definition of the Gorenstein flat modules. But $I_{S} \otimes_{S}\left(P_{R} \otimes F_{i}\right) \cong\left(I_{S} \otimes_{S} P_{R}\right) \otimes_{R} F_{i}$; hence (8) is a $\left(I_{S} \otimes-\right)$ exact exact sequence for any injective $S$-module. So ${ }_{S} P \otimes_{R} F$ is Gorenstein flat. Thus ${ }_{S} P_{R}$ is a Gorenstein flator.

From the last proposition we know that under some conditions a flator is a $G$-flator. Next we shall consider the problem under what conditions a $G$-flator is a flator.

Proposition 3.2. Let $R$ be a Gorenstein ring and let ${ }_{S} P_{R}$ be an $(R, S)$ bimodule with ${ }_{S} P$ projective and $P_{R}$ finitely generated projective. If $P_{R}$ is a G-flator, then $P_{R}$ is a flator.

Proof. Suppose that ${ }_{R} F$ is a flat left $R$-module. Then it is a Gorenstein flat module. If ${ }_{S} P_{R}$ is a $G$-flator, then ${ }_{S} P_{R} \otimes_{R} F$ is a Gorenstein flat left $S$-module. Since ${ }_{S} P_{R}$ is left $S$-projective, it has finite injective dimension by [7]. Assume 
that $i d\left({ }_{S} P\right) \leq n$. By the proof of Lemma 2.2, we know that ${ }_{S} P_{R} \otimes_{R} F$ has the finite injective dimension. Then the flat dimension of ${ }_{S} P_{R} \otimes_{R} F$ is also finite. By [7, Corollary 10.3.4], the flat dimension of ${ }_{S} P_{R} \otimes_{R} F$ is 0 . So ${ }_{S} P_{R} \otimes_{R} F$ is flat. Thus $P_{R}$ is a flator.

Bennis defined the strongly Gorenstein flat modules in [3]. Next, we shall use the strongly Gorenstein modules to character the Gorenstein flator.

Lemma 3.3. Let $R$ be a right coherent ring. Then a module is Gorenstein flat if and only if it is a direct summand of strongly Gorenstein flat modules.

Proof. The direct implication is immediately from [3, Theorem 3.5]. For the converse implication, it sufficient to prove that a direct summand of the strongly Gorenstein flat modules is a Gorenstein flat module. By [9, Theorem 3.7] we know that the Gorenstein flat modules closed under direct summand. However, a strongly Gorenstein flat module is a Gorenstein flat module. So we get the result.

Definition 3.4 (Gorenstein flat dimension). As done in [8] (and similar to the Gorenstein projective case), we define the Gorenstein flat dimension, $G f d_{R} M$, of a module $M$ by declaring that $G f d_{R} M \leq n$ if and only if $M$ has a resolution of Gorenstein flat modules of length $n$.

Lemma 3.5. Let $R$ be a Gorenstein ring. Then an $R$-module $M$ is strongly Gorenstein flat if and only if there exists a short exact sequence $0 \rightarrow_{R} M \rightarrow_{R}$ $F \rightarrow_{R} M \rightarrow 0$, where $F$ is a flat $R$-module.

Proof. $(\Rightarrow)$ It is straightforward.

$(\Leftarrow)$ Since $R$ is a Gorenstein ring, it is a Notherian ring. By [7, Theorem 12.3.1], $G f d_{R} M \leq \infty$ for all $R$-modules $M$. Assume $G f d_{R} M \leq n$. Then $\operatorname{Tor}_{R}^{n+1}(M, X)=0$ for all $X$ with finite injective dimension. Furthermore, by the dual case of [4, Proposition 3.15] (it is also right under noncommutative condition), we know that $M$ is strongly Gorenstein flat.

Lemma 3.6. Let $R$ be a Gorenstein ring and let ${ }_{S} P_{R}$ be a flator with ${ }_{S} P$ is a projective left $S$-module. Then ${ }_{S} P_{R}$ is a G-flator.

Proof. First, we shall prove that ${ }_{S} P_{R} \otimes_{R}-$ preserves strongly Gorenstein flat left $R$-modules. Suppose that $M$ is a strongly Gorenstein flat left $R$-module, by Lemma 3.5 there exists a short exact sequence $0 \rightarrow_{R} M \rightarrow_{R} F \rightarrow_{R} M \rightarrow 0$ of left $R$-modules, where $F$ is a flat left $R$-module. Applying ${ }_{S} P_{R} \otimes_{R}-$ to the above exact sequence, we have the following exact sequence

$$
0 \rightarrow S P_{R} \otimes_{R} M \rightarrow_{S} P_{R} \otimes_{R} F \rightarrow_{S} P_{R} \otimes_{R} M \rightarrow 0,
$$

where ${ }_{S} P_{R} \otimes_{R} F$ is a flat left $S$-module. By Lemmas 2.2 and 3.5 we know that ${ }_{S} P_{R} \otimes_{R} M$ is strongly Gorenstein flat.

Now, we shall show ${ }_{S} P_{R} \otimes_{R}-$ preserves Gorenstein flat left $R$-modules. Suppose that $M$ is Gorenstein flat. Then by Lemma 3.3, there exists a left 
$R$-module $Q$ and a strongly Gorenstein flat left $R$-module $G$ such that ${ }_{R} G \cong_{R}$ $M \bigoplus_{R} Q$. Applying ${ }_{S} P_{R} \otimes_{R}-$ to the above formula we get ${ }_{S} P_{R} \otimes_{R} G \cong \cong_{S}$ $P_{R} \otimes_{R} M \bigoplus_{S} P_{R} \otimes_{R} Q$. Note that ${ }_{S} P_{R} \otimes_{R} G$ is a strongly Gorenstein flat left $S$-module, so ${ }_{S} P_{R} \otimes_{R} M$ is a Gorenstein flat left $S$-module by Lemma 3.3.

Theorem 3.7. Let $R$ be a Gorenstein ring, and let ${ }_{S} P_{R}$ be an $(R, S)$-bimodule with ${ }_{S} P$ projective and $P_{R}$ finitely generated projective. Then $P_{R}$ is a flator if and only if $P_{R}$ is a $G$-flator.

Proof. $(\Leftarrow)$ Following Proposition 3.2.

$(\Rightarrow)$ By the proof of Lemma 3.6.

Corollary 3.8. Let ${ }_{S} P_{R}$ be a finitely generated projective left $R$-module. If ${ }_{S} P_{R} \otimes_{R}-$ preserves strongly Gorenstein flat left $R$-modules, then ${ }_{S} P_{R}$ is a $G$-flator.

Following [9], we know that there are some relations between Gorenstein flat modules and strongly copure flat modules.

Proposition 3.9. Let $P_{R}$ be a flator and ${ }_{S} P_{R} \otimes_{R} F$ be a copure flat left $S$ module for any Gorenstein flat $R$-module $F$. Then $P_{R}$ is a G-flator.

Proof. Assume that $F$ is a Gorenstein flat left $R$-module. So there exists an $(\mathcal{I}(R) \otimes-)$-exact exact sequence

$$
\cdots \rightarrow_{R} F_{1} \rightarrow_{R} F_{0} \rightarrow_{R} F^{0} \rightarrow_{R} F^{1} \rightarrow \cdots
$$

of flat $R$-modules such that $F=\operatorname{Ker}\left(F^{0} \rightarrow F^{1}\right)$. Applying ${ }_{S} P_{R} \otimes-$ to this exact sequence, we get the following exact sequence of copure flat $S$-modules

$$
\cdots \rightarrow_{S} P_{R} \otimes_{R} F_{1} \rightarrow_{S} P_{R} \otimes_{R} F_{0} \rightarrow_{S} P_{R} \otimes_{R} F^{0} \rightarrow_{S} P_{R} \otimes_{R} F^{1} \rightarrow \cdots,
$$

where ${ }_{S} P_{R} \otimes_{R} F=\operatorname{Ker}\left({ }_{S} P_{R} \otimes_{R} F^{0} \rightarrow_{S} P_{R} \otimes_{R} F^{1}\right)$. By using the hypothesis for any injective right $S$-module $E_{S}$, the functor $E_{S} \otimes-$ makes the above exact sequence exact. Thus ${ }_{S} P_{R} \otimes_{R} M$ is Gorenstein flat.

Proposition 3.10. Let $P_{R}$ be a flator and ${ }_{S} P_{R} \otimes_{R} F$ be a copure flat for any Gorenstein flat $R$-module $F$. If ${ }_{R} N \in \widehat{\mathcal{G \mathcal { F }}(R)}$, then ${ }_{S} P_{R} \otimes_{R} N \in \widetilde{\mathcal{G F}(S)}$.

Proof. We proceed by induction on $\operatorname{Ffd}_{R}(N)=n$. If $n=0$, then $N$ is Gorenstein flat. Hence the result follows by Proposition 3.9. Now suppose inductively that the result has been proved for all values smaller than $n$, and so we prove it for $n$. As $G f d_{R}(N)=n$, there is an exact sequence $0 \rightarrow C \rightarrow M \rightarrow N \rightarrow 0$ of $R$-modules such that $M$ is Gorenstein flat and $\operatorname{Gid}_{R}(C) \leq n-1$ by [9, Proposition 2.18]. Applying ${ }_{S} P_{R} \otimes_{R}-$ to the above exact sequence, we get the following exact sequence of $S$-modules.

$$
0 \rightarrow{ }_{S} P_{R} \otimes_{R} C \rightarrow_{S} P_{R} \otimes_{R} M \rightarrow{ }_{S} P_{R} \otimes_{R} N \rightarrow 0 .
$$

Then we can use the induction hypothesis for $C$ and conclude that ${ }_{S} P_{R} \otimes_{R} C$ has finite Gorenstein flat dimension. By Proposition 3.9 the left $S$-module 
${ }_{S} P_{R} \otimes M$ is Gorenstein flat. Then the proceeding exact sequence implies that ${ }_{S} P_{R} \otimes N$ has finite Gorenstein flat dimension.

Corollary 3.11. Let ${ }_{S} P_{R}$ be a finitely generated projective left $R$-module, and ${ }_{S} P_{R}$ be a G-flator. If a left $R$-module $N \in \widetilde{\mathcal{G F}}(R)$, then the left $S$-module ${ }_{S} P_{R} \otimes_{R} N \in \widetilde{\mathcal{G F}}(S)$.

Next, we shall study the relations between the $G$-flator and the Gorenstein flat dimension of the left $R$-modules.

Theorem 3.12. Let $P_{R}$ be a finitely generated projective $R$-module. Then $P_{R}$ is a $G$-flator if and only if $G f d\left(P_{R} \otimes_{R} M\right) \leq G f d\left({ }_{R} M\right)$.

Proof. $(\Longrightarrow)$ Let $M$ be a left $R$-module. Then $M$ has a Gorenstein flat resolution:

$$
\cdots \rightarrow_{R} F_{2} \rightarrow_{R} F_{1} \rightarrow_{R} F_{0} \rightarrow M \rightarrow 0 .
$$

Applying ${ }_{S} P_{R} \otimes-$ to this resolution, we get the following exact sequence:

$$
\cdots \rightarrow{ }_{S} P_{R} \otimes_{R} F_{2} \rightarrow_{S} P_{R} \otimes_{R} F_{1} \rightarrow_{S} P_{R} \otimes_{R} F_{0} \rightarrow_{S} P_{R} \otimes M \rightarrow 0 .
$$

Where each ${ }_{S} P_{R} \otimes_{R} F_{i} \quad(i \geq 0)$ is a Gorenstein flat left $S$-module as $P_{R}$ is a $G$-flator. So $G f d_{S}\left({ }_{S} P_{R} \otimes_{R} M\right) \leq G f d_{R}\left({ }_{R} M\right)$.

$(\Longleftarrow)$ Suppose that $G f d_{S}\left({ }_{S} P_{R} \otimes_{R} M\right) \leq G f d_{R}\left({ }_{R} M\right)$. Let $M$ be a Gorenstein flat left $R$-module. Then $\operatorname{Gifd}_{R}\left({ }_{R} M\right)=0$, so $\operatorname{Gfd}_{S}\left({ }_{S} P_{R} \otimes_{R} M\right)=0$, and thus ${ }_{S} P_{R} \otimes_{R} M$ is a Gorenstein flat module. Therefore, ${ }_{S} P_{R}$ is a Gorenstein flator.

Definition 3.13. We call $P_{R}$ a perfect $G$-flator if ${ }_{S} P_{R}$ preserves Gorenstein flat precovers, that is, if $G \rightarrow M$ is a Gorenstein flat precover of $M$, then ${ }_{S} P_{R} \otimes_{R} G \rightarrow P_{R} \otimes_{R} M$ is also a Gorenstein flat precover of $P_{R} \otimes_{R} M$.

Theorem 3.14. Let $R$ be a Gorenstein ring, and let ${ }_{S} P_{R}$ be an injector with ${ }_{S} P$ projective and $-\otimes_{R} P_{S}^{*}$ preserve injective modules. Then $P_{R}$ is a flator if and only if $P_{R}$ is a perfect $G$-flator.

Proof. Similar to the proof of Theorem 2.14, it is easy to see that if $P_{R}$ is a flator, then it is a $G$-flator. First, we shall prove ${ }_{R} P_{S}^{*} \otimes_{S}-$ preserves Gorenstein flat modules. For any Gorenstein flat module ${ }_{S} M$, by the definition of Gorenstein flat modules, there exists a $J_{S} \otimes_{S}$-exact complete flat resolution of flat modules (where $J_{S}$ is an arbitrary injective right $S$-module)

$$
\mathbb{F}=\cdots \rightarrow_{S} F_{1} \rightarrow_{S} F_{0} \rightarrow_{S} F^{0} \rightarrow_{S} F^{1} \rightarrow \cdots
$$

such that ${ }_{S} M \cong \operatorname{Ker}\left({ }_{S} F^{0} \rightarrow{ }_{S} F^{1}\right)$. Applying ${ }_{R} P_{S}^{*} \otimes_{S}-$ to (9), we get a sequence of left $R$-modules

(10) $\quad \cdots \rightarrow_{R} P_{S}^{*} \otimes_{S} F_{1} \rightarrow_{R} P_{S}^{*} \otimes_{S} F_{0} \rightarrow_{R} P_{S}^{*} \otimes_{S} F^{0} \rightarrow_{R} P_{S}^{*} \otimes_{S} F^{1} \rightarrow \cdots$.

Since $P_{R}$ is an injector, by [1, Theorem 2.1], ${ }_{R} P_{S}^{*}$ is right $S$-flat and left $R$ projective. So (9) is an exact sequence of flat left $R$-modules. Moreover,

$$
{ }_{R} P_{S}^{*} \otimes_{S} M \cong \operatorname{Ker}\left({ }_{R} P_{S}^{*} \otimes_{S} F_{0} \rightarrow{ }_{R} P_{S}^{*} \otimes_{S} F^{0}\right) .
$$


For each injective $R$-module $I_{R}$, by the supposition $I_{R} \otimes_{R} P^{*}$ is an injective right $S$-module. So $\left(I_{R} \otimes_{R} P^{*}\right) \otimes \mathbb{F}$ is exact. Note that

$$
I_{R} \otimes_{R}\left(P_{S}^{*} \otimes_{S} F_{i}\right) \cong\left(I_{R} \otimes_{R} P_{S}^{*}\right) \otimes_{S} F_{i}, \quad i \geq 0
$$

for any right $R$-module $I_{R}$, then $(10)$ is an $\left(I_{R} \otimes-\right)$-exact exact sequence. Hence ${ }_{R} P_{S}^{*}$ preserves Gorenstein flat modules.

Suppose that ${ }_{R} G \rightarrow_{R} M$ is a Gorenstein flat precover of left $R$-module $M$. Since $P_{R}$ is a Gorenstein flator, ${ }_{S} P_{R} \otimes_{R} G$ is Gorenstein flat. Using the same method in the proof of Theorem 2.14 and the definition of the Gorenstein flat precover, we can get the result.

Proposition 3.15. Let $R$ be a quasi-Frobenius ring, and let ${ }_{R} P_{S}$ be an $(R, S)$ bimodule with ${ }_{S} P$ projective and $P_{R}$ finitely generated projective. Then the following are equivalent.

(1) $P_{R}$ is a Gorenstein injector.

(2) $P_{R}$ is a Gorenstein projector.

(3) $P_{R}$ is a Gorenstein flator.

Proof. We only prove $(1) \Rightarrow(2),(2) \Rightarrow(3)$ and $(3) \Rightarrow(1)$ are similarly.

Let $M$ be a Gorenstein injective right $R$-module, $M$ is also Gorenstein projective by $\left[7, P_{257}\right.$ Exercise 5]. Since $P_{R}$ is a Gorenstein injector, ${ }_{S} P_{R} \otimes$ $M$ is Gorenstein injective. $S=\operatorname{End}\left(P_{R}\right)$ is also a quasi-Frobenius ring by Lemma 2.2, so ${ }_{S} P_{R} \otimes M$ is Gorenstein projective by [7, $P_{257}$ Exercise 5].

Acknowledgements. This work was partially supported by the Natural science Foundation of Jiangsu province of China (BK 2007133). The authors would like to give many thanks to the referee for his or her useful suggestions and comments, which have improved this paper.

\section{References}

[1] F. W. Anderson, Endomorphism rings of projective modules, Math. Z. 111 (1969), 322332 .

[2] F. W. Anderson and K. R. Fuller, Rings and Categories of Modules, Graduate Texts in Mathematics, Vol. 13. Springer-Verlag, New York-Heidelberg, 1974.

[3] D. Bennis and N. Mahdou, Strongly Gorenstein projective, injective, and flat modules, J. Pure Appl. Algebra 210 (2007), no. 2, 437-445.

[4] _ Global Gorenstein dimension, Proc. Amer. Math. Soc. 138 (2010), no. 2, 461465.

[5] E. E. Enochs and O. M. G. Jenda, Copure injective resolutions, flat resolvents and dimensions, Comment. Math. Univ. Carolin. 34 (1993), no. 2, 203-211.

[6] __ Gorenstein injective and projective modules, Math. Z. 220 (1995), no. 4, 611633.

[7] _ Relative Homological Algebra, de Gruyter Expositions in Mathematics, 30. Walter de Gruyter \& Co., Berlin, 2000.

[8] E. E. Enochs, O. M. G. Jenda, and B. Torrecillas, Gorenstein flat modules, Nanjing Daxue Xuebao Shuxue Bannian Kan 10 (1993), no. 1, 1-9.

[9] H. Holm, Gorenstein homological dimensions, J. Pure Appl. Algebra 189 (2004), no. $1-3,167-193$. 
[10] R. W. Miller, Endomorphism rings of finitely generated projective modules, Pacific J. Math. 47 (1973), 199-220.

[11] K. Morita, Adjoint pairs of functors and Frobenius extensions, Sci. Rep. Tokyo Kyoiku Daigaku Sect. A 9 (1965), 40-71.

[12] J. J. Rotman, An Introduction to Homological Algebra, Pure and Applied Mathematics, 85. Academic Press, Inc. [Harcourt Brace Jovanovich, Publishers], New York-London, 1979.

[13] J. Xu, Flat Covers of Modules, Lecture Notes in Mathematics, 1634. Springer-Verlag, Berlin, 1996.

QINQIN GU

School of Mathematics And Physics

Anhui University of Technology

MaAnshan, P. R. China

E-mail address: gqq0634@163.com

Xiaosheng Zhu

Department of Mathematics

NANJING UNIVERSITY

NAnjing, P. R. China

E-mail address: zhuxs@nju.edu.cn

WenPING ZhOU

School of Mathematics and Physics

AnHui University of TECHNOLOGY

Maanshan, P. R. China

E-mail address: luobei666@126.com 